\title{
The Research and Design of Multi - functional Family Assistant Based on Embedded System
}

\author{
Yongqi Han ${ }^{1, a, *}$, Pei Xiao ${ }^{1, b}$, Bei Zhu ${ }^{1, c}$ and Yan $\mathrm{Li}^{1, \mathrm{~d}}$ \\ ${ }^{1}$ Jilin Agricultural University College of Information Technology, Changchun, 130118, China \\ a yongqihan@163.com, ${ }^{b} 970076933 @ q q . c o m,{ }^{c} 549056797 @ q q . c o m,{ }^{d} 287199716 @ q q . c o m$ \\ ${ }^{*}$ corresponding author
}

Keywords: Embedded, Smart home, STC12C5A60S2, Multi-functional, Family assistant, Smart dolls

Abstract. Based on the embedded multi - functional family assistant is a smart robot doll based on the Internet of Things with multiple operating modes, which is composed of many functions, such as, calendar, weather forecast, indoor temperature and humidity, memorandum, an alarm system for matter safety supervision and the like, making human life more interesting.

\section{Introduction}

The Multi-functional Family Assistant designed in this article is an interesting smart swing doll basing on the functions of calendar in the Internet of Things, weather forecast, temperature and humidity indoor, memorandum, an alarm system for matter safety supervision and the like. It belongs to a part of smart home and could provide convenience to people's daily life.

\section{System Structure and Function Overview}

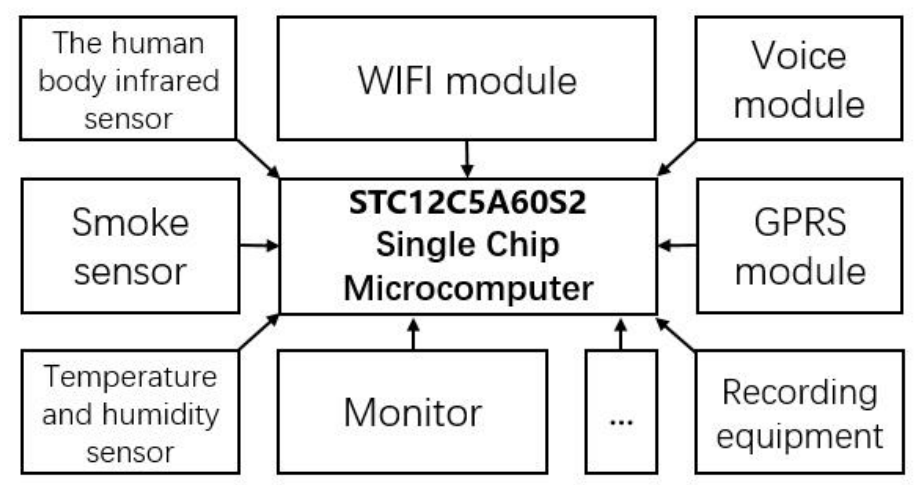

Figure $1 \mathrm{~A}$ whole structure diagram of the system

From Figure 1 we can see that multi-functional family assistant takes the STC12C5A60S2 as main control chip, which is composed of infrared sensor, Wireless Fidelity, smog sensor, temperature and humidity sensor, GPS modules etc.

Through the WIFI module to send and receive relevant data; Temperature and humidity indoor can be obtained through temperature and humidity module; Displayer is used for displaying information like weather conditions of the day, the time now, temperature and humidity, which is convenient for users to view at any time; Moreover, voice module can broadcast messages corresponding to the displayer.

As shown in the figure 2, the system provides two kinds of modules, general module and fortified module for switching. Both general module and fortified module have smoke alarm function. When users are at home, they could turn on the general module that has functions of on time alarm, dressing reminder, voice memo, music player, etc. Besides, when users go out, they could turn on fortified module. When switching from general module to fortified module, assistant will offer related recommendations about user's trip on the basis of weather information; For example, according to temperature giving users recommendations on dressing and reminding users 
taking umbrellas if rains, etc. Finally, the assistant would remind the user that whether they have taken objects like keys, phones, etc. (travel objects could be modified voluntarily through mobile App.) Under the fortified module, the assistant has functions like intelligent security, short message alarm, and short message memorandum, which enables users to work and playing at ease.

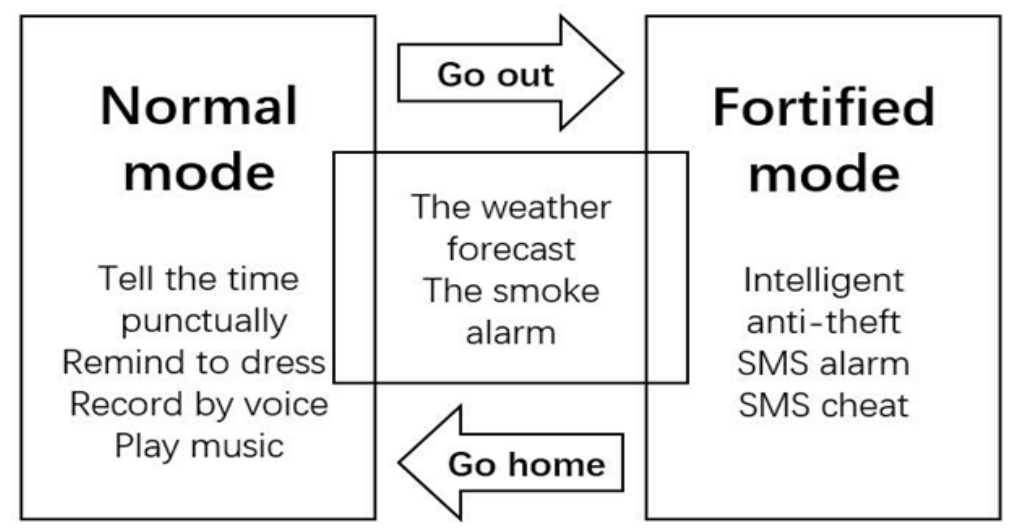

\section{Hardware System Design}

Figure 2 System functions diagram

\section{1) STC12C5A60S2 Single Chip Microcomputer}

The series of STC12C5A60S2 Single Chip Microcomputer is a single machine with single clock / machine cycle(1T) produced by Hong Jing Company, which is the newest high-speed, lower-power and strong anti-interference single-chip microcontroller. Its instruction code is compatible with traditional 8051 totally.

\section{2) ATK-ESP8266 WIFI Module}

ATK-ESP8266 is a kind of high-performance UART-Wireless Fidelity module launched by ALIENTEK and this module not only supports LVTTL UART but also is compatible with 3.3V \& $5 \mathrm{~V}$ single chip microcomputer system, which will be convenient for connecting with STC12C5A60S2 SCM.

ATK_ESP8266 supports three modes, STA/AP/STA+AP. What the system adopts is STA+AP mode; In this mode, module can be taken as both Wireless Fidelity AP and Wireless Fidelity STA; Moreover, other Wireless Fidelity devices could connect with the module, so could the module link other Wireless Fidelity.

\section{3) SYN6658 Chinese Speech Synthesis Module}

SYN6658 Chinese Speech Synthesis Module is a high-end speech synthesis chip launched by Yuying World Technology Company of Beijing, which is famous for higher price ratio and more natural effect. YN6658 receives textual data remained to be compounded through communication methods of UART port or SPI port, realizing the conversion from text to audio (TTS audio).

\section{4) Other Modules And Their Corresponding Functions}

The normal operation of the entire system, but also need to work together with other module.

Here are introductions about other modules and functions of the whole system:

- HC-SR501 Body Sensor Module is applied to the intelligent anti-theft function of fortified mode;

- MQ-2 Smoke and Gas Sensor Module is used for smoke alarm function;

- SIM900A GPRS module can send related short messages to mobile phones that the users have set up;

- LCD12864 Displayer is used for displaying corresponding information;

- DHT-11 Temperature and Humidity module is used for detecting temperature and humidity indoor;

- ISD1820 Record Playback is used for voice memos;

- DS1302 Clock is used for system clock.

\section{Software System Design}

"Family_ Assistant. c" is a document in which the main function exists and in this document, the main function implements each function of the system by calling functions in options of 
“DS1302.c”、“LCD12864.c”、“DHT-11.c”、“KEY.c”、“SIM900A.c”、“ESP8266.c”、“6658.c”.Here is a brief introduction of partial function and their functions in the $\mathrm{C}$ document:

The flow chart of main function, as shown in Figure 3, to begin with, function would initialize the system. Then, it will execute different cyclic operation in accordance with different modes.

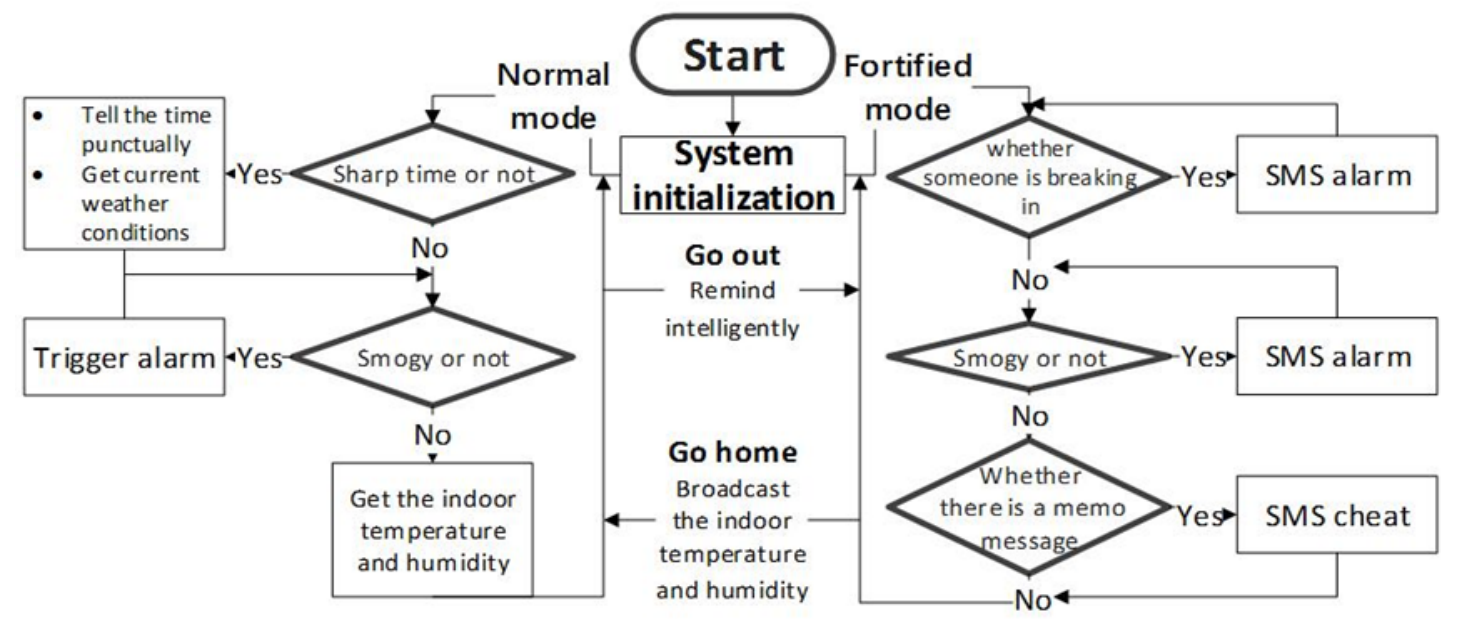

Figure 3 The flow chart of main function

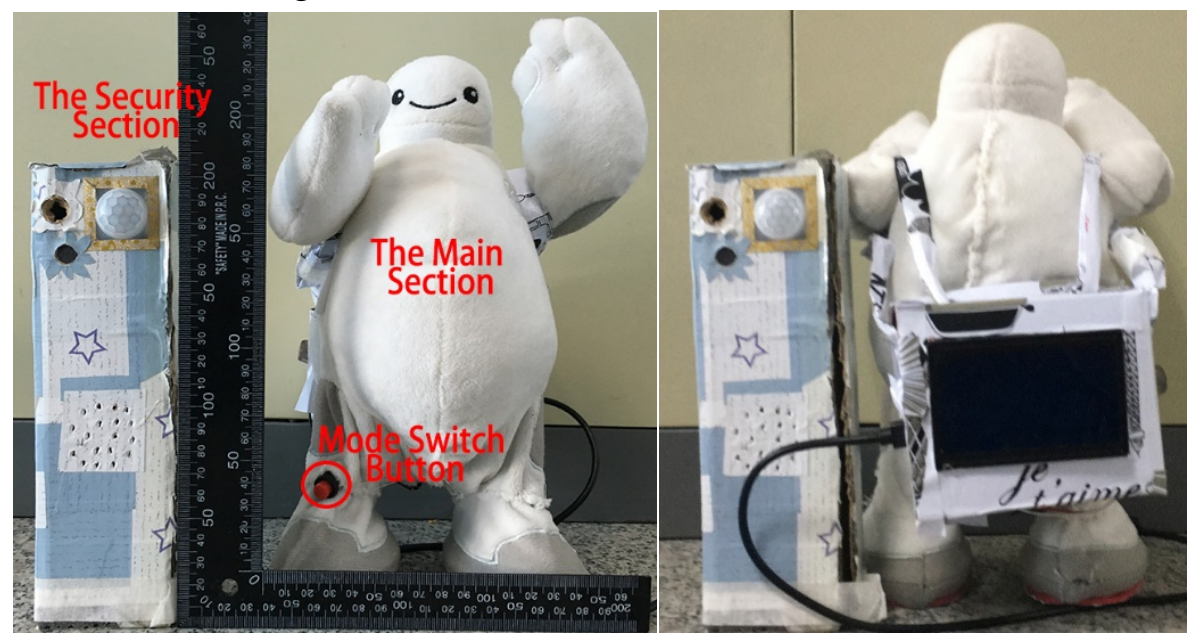

Figure 4 The physical map of multi-functional family assistant(pros and cons)

\section{Experimental Product Test Results}

\section{Experimental Product Introduction}

The two pictures in Figure 4 are effect pictures taken from pros and cons of the multi-functional family assistant. The first picture is front view of the assistant, from which we can see that the whole assistant consists of two sections, the main section and the security section. The main section of assistant is a swing doll (Around $200 \mathrm{~mm} \times 180 \mathrm{~mm} \times 250 \mathrm{~mm}$ ); Besides, there is a switch button of mode on the right leg for realizing one keyboard toggle. However, the security section (Around $50 \mathrm{~mm} \times 30 \mathrm{~mm} \times 200 \mathrm{~m}$ ) can be applied to detecting whether there were thieves breaking in or not. The second picture is back view of the assistant.

\section{Build A Test Environment}

In the test, we put the main section of assistant on experiment table and the security section next to the main section. Because there is no available test server, we use laptop computer to construct corresponding test server; Use android phones running WIFI test software.

\section{Test Results}

In normal mode, we use the assistant to achieve the weather forecast, punctuality, playing music, smoke alarm and voice memo and other functions. When we switch to security mode, the assistant will voice the relevant travel advice. In the security mode, we will SMS alarm time set to 15 
seconds, that is, continuous 15 seconds detection someone is there, it will touch the SMS alarm, and the doll will always swing, set up SMS alarm time is in order to leave buffer time for users to switch to normal mode, in this mode, in addition to SMS alarm, but also to achieve a message memo, SMS smoke alarm and other functions. When switching to normal mode, the assistant will broadcast the current room temperature and humidity. And the use of mobile phones on the WIFI test software, send the relevant instructions to achieve a remote mode switch and other functions.

\section{Conclusion}

After the experimental product test, Although the assistant can complete the design of the relevant functions, but there are still many places need to be improved. The first part need to improve is the main part of the connection with the anti-theft part. the connection using wired connections, which is not conducive to our full monitoring of indoor conditions; We propose the program is the use of Zigbee ad hoc network technology, set up a number of anti-theft part, which can achieve indoor dead angle monitoring, greatly improving the security performance of the assistant. And then the appearance of the assistant needs to be improved, the appearance of the assistant using manual design, not beautiful, While its LCD display is a form of backpack placed in the back of the assistant is not conducive to the user to view the relevant information, And the display screen is too monotonous, For the overall appearance, We will put the monitor in front and redesign the display part, then design the corresponding model, and next use the $3 \mathrm{D}$ printer to print out, so as to make up for its lack of design. Finally, the production of mobile APP, because at this stage is the use of WIFI test software to send instructions to complete the operation is not conducive to user operation, so in order to improve the user experience, we will design the corresponding mobile APP.

\section{Acknowledgments}

This work was funded by 2015 national college students innovation and entrepreneurship training program, 2015 Jilin college students innovation and entrepreneurship training program-Multifunctional Family Assistant.

\section{References}

[1] Krolewski J, Nowak D, Groblicka A, et al. INTERACTIVE SMART DOLL:, WO/2016/032835[P]. 2016.

[2] Singh P, Wang G, Cheverton M A, et al. Method and system for multi-functional embedded sensors[J]. 2017.

[3] Kim B J, Noh Y H, Jeong D U. Implementation of Mobile Based Multi-Function Health Monitoring System[C]// International Conference on It Convergence and Security. 2013:1-4.

[4] Guoqing J, Peng J. Portable and user-friendly multi-functional thermos cup[J]. 2016.

[5] Han Y, Zhao X, Li M, et al. Design of Intelligent Classroom Bell System Based on Internet of Things[C]// International Conference on Information Engineering for Mechanics and Materials. 2015. 